Please do not remove this page

RMIT

UNIVERSITY

\title{
A Depolarized Dynamic Light Scattering Method to Calculate Translational and Rotational Diffusion Coefficients of Nanorods
}

Nixon-Luke, Reece; Bryant, Gary

https://researchrepository.rmit.edu.au/esploro/outputs/9921863398101341/filesAndLinks?institution=61RMIT_INST\&index=null

Nixon-Luke, R., \& Bryant, G. (2019). A Depolarized Dynamic Light Scattering Method to Calculate Translational and Rotational Diffusion Coefficients of Nanorods. Particle and Particle Systems Characterization, 36(2), 1-7. https://doi.org/10.1002/ppsc.201800388

Document Version: Accepted Manuscript

Published Version: https://doi.org/10.1002/ppsc.201800388

Repository homepage: https://researchrepository.rmit.edu.au

(c) 2018 WILEY-VCH Verlag GmbH \& Co. KGaA, Weinheim

Downloaded On 2023/04/26 22:55:40 +1000 


\title{
A Depolarized Dynamic Light Scattering Method to Calculate Translational and Rotational Diffusion Coefficients of Nanorods
}

\author{
Reece Nixon-Luke and Gary Bryant* \\ Centre for Molecular and Nanoscale Physics, School of Applied Sciences, RMIT University, \\ Melbourne, Victoria 3001, Australia \\ E-mail: reece.nixon-luke@rmit.edu.au
}

Phone: +6139925 1245

\begin{abstract}
We present a new analysis of depolarized dynamic light scattering data which allows the unambiguous determination of rotational and translational diffusions coefficients of nanorods in suspension. By visualizing data scaling, purely translational diffusive motions can be isolated from vertically polarized scattering, allowing the unique determination of rotational diffusion from the depolarized scattering. We apply the method to nanorods with four different aspect ratios, and compare with theoretical predictions. Diffusion coefficients obtained show good agreement with calculations based on the direct measurements of rod length and diameter. Where the theories are shown to be valid, the method allows the measurement of statistically meaningful particle sizes and aspect ratios.
\end{abstract}




\section{Introduction}

Dynamic Light Scattering (DLS) provides information on the diffusivity of colloidal particles via analysis of the electric field autocorrelation function (EACF). Extraction of particle size may be done using Cumulant expansion or Laplace inversion methods, whereby the latter attempts to obtain the full particle size distribution (PSD). These models work best when describing spherical scatterers, where no contribution from rotational diffusion is present in the EACF.

Synthesis and characterisation of non-spherical nanoparticles has increased in recent years due to their interesting optical properties ${ }^{1}$ and their potential in biomedical applications. ${ }^{2}$

Depolarized dynamic light scattering (DDLS) is a viable method for characterising nonspherical particles, and has been applied to optically anisotopic particles such as the tobacco mosaic virus ${ }^{3}$ gold nanorods, ${ }^{4}$ spheres with symmetric optical anisotropy, ${ }^{5-7}$ carbon nanotubes ${ }^{8}$ and cellulose nanocrystals. ${ }^{9}$ Recently, DDLS has also been used for studying more subtle anisotropy effects. ${ }^{10,11}$ Despite this, DDLS remains challenging as data processing requires several assumptions that have not been thoroughly tested across a range of particle sizes, shapes, and materials. The interpretation is also complicated by the low depolarized signal, and angular dependent coupling between translational and rotational Brownian motion. ${ }^{12}$

Theory suggests that for long rods whose diameter is small compared to the illuminating wavelength, the rotational contribution to the EACF can be calculated as a function of scattering angle. ${ }^{13}$ According to this theory, at low scattering vectors $(q)$ the ratio of translational to rotational diffusion $>>1$ and therefore the EACF should form a single exponential decay. However, this theory does not take into account optical anisotropy of gold nanoparticles which has been shown to add a second decay mode from rods which, when considering only their geometric properties, should have only one. ${ }^{14}$ This raises questions of which diffusion coefficient this decay mode relates to, and how scattering angle influences this. 
The difficulty lies in determining the range of scattering angles whereby only translational motions make a significant contribution to the temporal decay of the EACF before applying models which make this assumption. In this article, we present a method to characterise rod like particles in an objective and unambiguous manner. We use the scaling of $\mathbf{q}^{2} \tau$ of the vertically polarized data to validate over which $\mathbf{q}$ range the assumptions are valid, and do this for both single and double decays. We then unambiguously determine the translational diffusion coefficient, and then use this as an input into the analysis of the horizontally polarized data to unambiguously determine the rotational diffusion coefficient.

\section{DLS Theory}

In DLS, the experimental quantity measured is the normalized intensity autocorrelation function $g^{(2)}(\mathbf{q}, \tau)$, where $\tau$ is the delay time. Under appropriate conditions, this can be used to calculate the EACF $\left(g^{(1)}(\mathbf{q}, \tau)\right)$ using the Siegert relation: ${ }^{15}$

$$
g^{(2)}(\tau)=1+\beta\left|g^{(1)}(\mathbf{q}, \tau)\right|^{2}
$$

where $\beta$ is an instrumental constant, and $\mathbf{q}$ is the scattering vector defined by:

$$
\mathbf{q}=\frac{4 \pi n}{\lambda} \sin \left(\frac{\theta}{2}\right)
$$

where $n$ is the refractive index of the medium, $\lambda$ is the wavelength of the laser and $\theta$ is the scattering angle.

$g^{(1)}(\mathbf{q}, \tau)$ is the quantity of interest as it is related to the particle displacement by:

$$
g^{(1)}(\mathbf{q}, \tau)=\frac{\left\langle A(\mathbf{q}, \tau) A^{*}(\mathbf{q}, 0) \exp (i \mathbf{q} \cdot \Delta \mathbf{r})\right\rangle}{\left\langle|A(\mathbf{q}, 0)|^{2}\right\rangle}
$$

where \langle\rangle denote a time average, $A(\mathbf{q})$ is the scattering amplitude of the particle, and $\Delta \mathbf{r}=$ $\mathbf{R}(\tau)-\mathbf{R}(0)$ describes the translational motion of the particle. For identical uniform particles, 
$A(\mathbf{q})=1$ so $g^{(1)}(\mathbf{q}, \tau)$ reads:

$$
g^{(1)}(\mathbf{q}, \tau)=\langle\exp (\mathrm{iq} \cdot \Delta \mathbf{r})\rangle=\exp (-\Gamma \tau)
$$

where $\Gamma$ is the decay rate:

$$
\Gamma=D_{t} \mathbf{q}^{2}
$$

where $D_{t}$ is the translational diffusion coefficient.

In practise, to fit the data we rearrange the Siegert relation:

$$
\sqrt{g^{(2)}(\tau)-1}=\sqrt{\beta} g^{(1)}(\mathbf{q}, \tau)
$$

to extract $g^{(1)}(\mathbf{q}, \tau)$ and the intercept $\sqrt{\beta}$.

Standard DLS typically uses a vertically polarized laser, and no polariser on the detector. By contrast standard DDLS introduces a polariser before the detector, and the scattered light is measured with the polariser in either a vertical $(V)$ or horizontal $(H)$ orientation - these are known as polarized $(V V)$ or depolarized $(V H)$ scattering respectively. We will use this terminology to refer to DLS data collected in these modes.

\section{Polarized DLS}

Standard DDLS analysis of rod-like particles involves obtaining an $V V$ angular dependant set of data at scattering angles low enough that rotational motions do not overly contribute to the decay of the EACF. A $V H$ data set is taken over the same range, whereby the contribution from the rotational motion is constant. Rotational diffusion coefficients can then be obtained by determining the intercept where $\theta=0$. The analysis works under the assumption that rotational information is not present in the $V V$ data over the range of scattering angles chosen.

Since conventional multi angle DLS instrumentation typically cannot reach scattering 
angles low enough to allow complete seperation of rotational and translational components, new schemes to measure rotational diffusion have been devised. The recent addition of near field scattering (NFS) techniques ${ }^{16}$ opened the door to custom built low angle light scattering (LALS) instruments. ${ }^{17}$ Rodlike particles are measured using a laser NFS set-up, ${ }^{18}$ where ultra low angle data was obtained allowing complete separation of the rotational and translational motions. A custom photon counting scheme ${ }^{19}$ was used to overcome the low depolarized scattering signal. A novel zero angle confocal DDLS instrument capable of processing samples with much higher turbidity that conventional DLS has been devised. ${ }^{20}$ These methods overcome some of the problems associated with traditional low angle light scattering, but require custom implementations, and the more common white light microscope based differential dynamic microscopy ${ }^{21}$ suffers from sensitivity issues with diffraction limited samples.

For rod-like samples, $V V$ contains information about the translational and rotational motions of a sample. The rotational component is described by the dynamic form factor, ${ }^{22}$ and is dependant on the aspect ratio (length (L) / diameter (D)) of the rods. For very long thin rods, where $\mathbf{q D}<<1$, the form factor is: ${ }^{23}$

$$
P(\mathbf{q}, \hat{u})=\left\langle j_{0}^{2}\left(\frac{1}{2} \mathbf{q} L \cdot \hat{u}\right)\right\rangle
$$

where $L$ is the length of the rod, $j_{0}$ is the spherical Bessel function of order 0 and $\hat{u}$ is the orientation of the rod.

when inserted into Eq. (3) the EACF becomes:

$$
g^{(1)}(\mathbf{q}, \tau)=\exp (-\Gamma \tau) \sum_{l=0}^{\infty} e^{-D_{r} l(l+1) \tau} S_{l}(\mathbf{q} L)
$$

where $S_{l}(\mathbf{q} L)$ is: 


$$
S_{2 l}(\mathbf{q} L)=\frac{(4 l+1)\left(\int_{-1}^{1} P_{2 l}(x) j_{0}\left(\frac{1}{2} \mathbf{q} L x\right) \mathrm{d} x\right)^{2}}{2 \int_{-1}^{1} j_{0}^{2}\left(\frac{1}{2} \mathbf{q} L x\right) \mathrm{d} x}
$$

where $P_{l}$ is a Legendre polynomial of order $l$.

Eq. 9 is the generalized function for determination of appropriate weights due to harmonic interference from scattering segments outwards from the center of a long and thin rod. ${ }^{24}$

The contribution to the EACF therefore takes the form of a sum of exponential decays of translational and rotational motions, whereby the weighting terms depend on $\mathbf{q} L$. The implications of Eq. (9) can be visualised when the expansion coefficients are plotted over a range of $\mathbf{q} L$ (Fig. 1). Practically, this indicates that in order to measure only $D_{t}$ from rod like particles, measurements must be made at small enough angles such that $\mathbf{q} L<5$, where the form factor is close to unity and the $S_{0}$ term dominates the signal. In this regime the EACF should take the form of Eq. 4. As scattering angles increase, higher order rotational motions enter the EACF which complicate the analysis. In the case of $5<\mathbf{q} L<10$, the EACF reads:

$$
g_{V V}^{(1)}(\mathbf{q}, \tau)=S_{0} \exp \left(-D_{t} \mathbf{q}^{2} \tau\right)+S_{2} \exp \left(-\left(D_{t} \mathbf{q}^{2}+6 D_{r}\right) \tau\right)
$$

\section{Depolarized DLS}

In DDLS, the intensity of depolarized light at the detector depends on two separate components: the phase caused by the centre of mass motion, and the amplitude caused by rotation of the particle. When depolarized scattering is measured, interpretation is easier as $D_{r}$ is a constant contribution across all scattering angles.

The depolarized correlation function takes the form: ${ }^{25}$ 


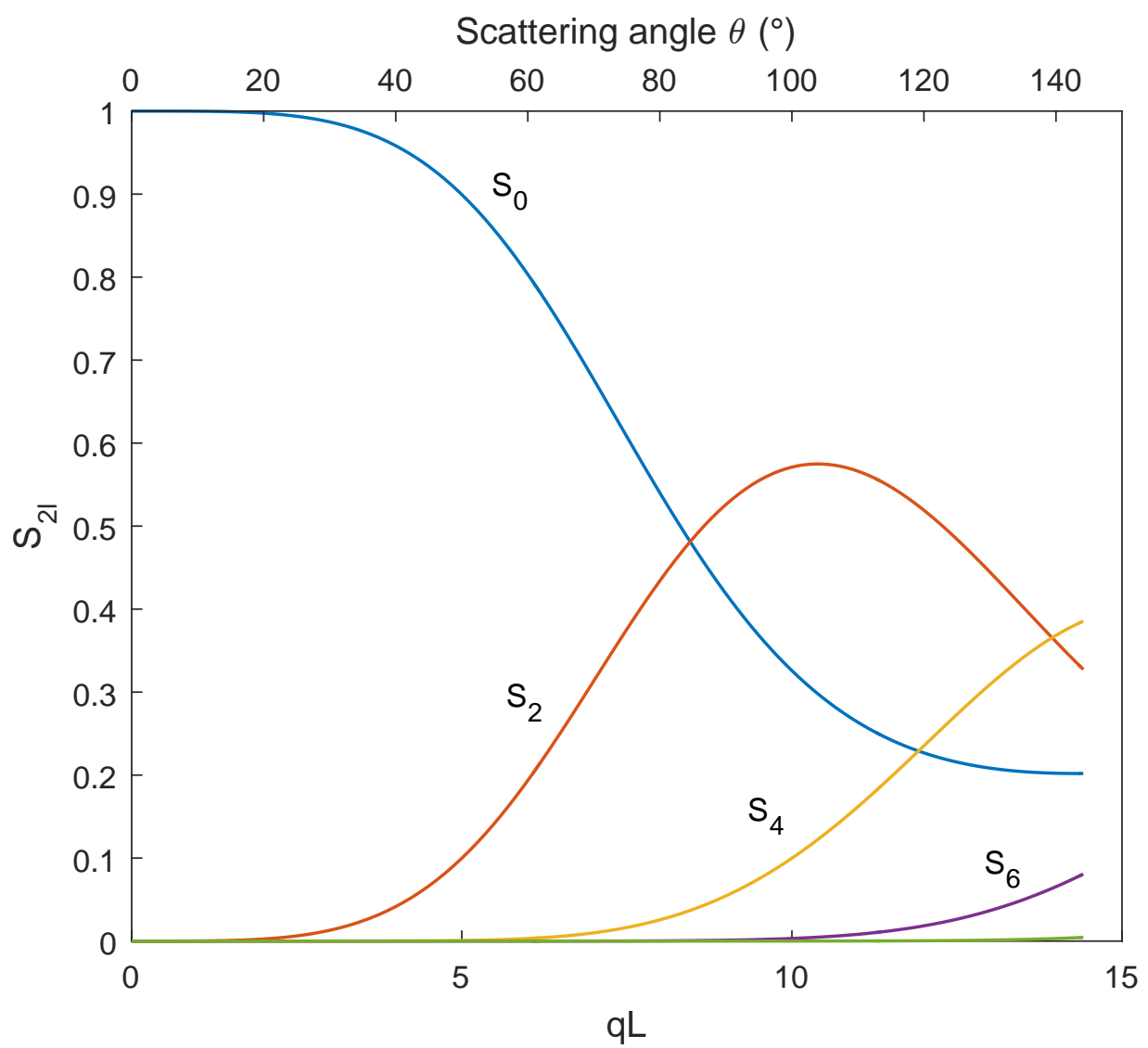

Figure 1: Calculated $S_{l}$ coefficients of Eq. (9) plotted as a function of $\mathbf{q} L$ (with scattering angle $\theta$ on the top axis), for a rod of length $\mathrm{L}=565 \mathrm{~nm}$ ) 


$$
g_{V H}^{(1)}(\mathbf{q}, \tau)=\sum_{m} \sum_{n}\left\langle P_{2}^{m}(\operatorname{vh}, \tau) P_{2}^{n}(\operatorname{vh}, 0) \exp (i \mathbf{q} \cdot \Delta \mathbf{r}\rangle\right)
$$

where $P_{2}^{m}$ is a Legendre Polynomial of order 2. The evaluation of Eq. (11) requires an explicit form of the ensemble averaged quantity $\left\langle P_{2}^{m}(\mathrm{vh}, \tau) P_{2}^{n}(\mathrm{vh}, 0)\right\rangle$ which depends on the motion of the body. For diffusion by a cylindrically symmetric ellipsoid the quantity has been evaluated as: ${ }^{13}$

$$
\left\langle P_{2}^{m}(\mathrm{vh}, \tau) P_{2}^{n}(\mathrm{vh}, 0)\right\rangle=\exp \left(-6 D_{r} \tau\right)
$$

The VH EACF is therefore:

$$
\left.g_{V H}^{(1)}(\mathbf{q}, \tau)=\exp \left(-\left(\mathbf{q}^{2} D_{t}+6 D_{r}\right) \tau\right)\right)
$$

Thus $D_{r}$ may be calculated as long as $D_{t}$ can be measured independently.

\section{Comparisons with theory}

In order to compare DDLS measured values with theory, we use relations that allow calculation of a theoretical $D_{t}$ and $D_{r}$ based on manufacturers measurmenets of $\mathrm{L}$ and $\mathrm{D}$ taken from TEM. It is noted that in the micrographs presented, size polydispersity is evident (see supplementary material Fig. S6). The Tirado and Garcia de la Torre (TG) ${ }^{26,27}$ theory is suggested to be applicable to rod like particles with an aspect ratio $p=L / D$ of $2<p<30$, whereas the Broersma relations ${ }^{28}$ are estimated to be valid in the range of $p>5$. We compare our results to both theories, although our rods have aspect ratios which lie within the range where the TG theory should be more applicable. Rod1.7 lies slightly out of the range for the TG theory, although it may still be suitable as it is not clear exactly where the theory breaks down and by how much. 
Tirado and Garcia de la Torre (TG) theory may be summarized in the following equations: ${ }^{26,27}$

$$
\begin{aligned}
& D_{t}=\frac{k_{\mathrm{B}} T}{3 \pi \eta L}\left(\ln \left(\frac{L}{D}\right)+\nu\right) \\
& \nu=0.312+0.565 \frac{D}{L}-0.1 \frac{D^{2}}{L^{2}}
\end{aligned}
$$

where $k_{\mathrm{B}}$ is Boltzmann's constant, $T$ is the absolute temperature, and $\eta$ is the solvent viscosity.

$$
\begin{aligned}
& D_{r}=\frac{3 k_{\mathrm{B}} T}{\pi \eta L^{3}}\left(\ln \left(\frac{L}{D}\right)+\sigma\right) \\
& \sigma=-0.662+0.917 \frac{D}{L}-0.05 \frac{D^{2}}{L^{2}}
\end{aligned}
$$

Broersma's relations (BR) may be summarised as: ${ }^{28}$

$$
\begin{gathered}
D_{t}=\frac{k_{\mathrm{B}} T}{3 \pi \eta L}\left(\delta-(1 / 2)\left(\gamma_{\perp}+\gamma_{\|}\right)\right) \\
\delta=\ln \left(\frac{2 L}{D}\right) \\
\gamma_{\|}=0.807+\frac{0.15}{\delta}+\frac{13.5}{\delta^{2}}-\frac{37}{\delta^{3}}+\frac{22}{\delta^{4}} \\
\gamma_{\perp}=-0.193+\frac{0.15}{\delta}+\frac{8.1}{\delta^{2}}-\frac{18}{\delta^{3}}+\frac{9}{\delta^{4}} \\
D_{r}=\frac{3 k_{\mathrm{B}} T}{\pi \eta L^{3}}(\delta-\xi) \\
\xi=1.14+\frac{0.2}{\delta}+\frac{16}{\delta^{2}}-\frac{63}{\delta^{3}}+\frac{62}{\delta^{4}} \\
\delta=\ln \left(\frac{2 L}{D}\right)
\end{gathered}
$$

\section{Scaling of the EACF}

An angular dependant set of EACFs can be checked for scaling by replotting each curve by the scaled time variable $\mathbf{q}^{2} \tau$. The existence of scaling indicates that detected motions 
conform to a diffusive type in accordance to $D_{t} \mathbf{q}^{2}$. When multiple decays are evident in the EACF, only the decay mode which corresponds to $D_{t}$ will scale (superimpose). By scaling the EACFs obtained from suspensions of rod like particles, it is possible to determine the range of scattering angles where decays due to $D_{t}$ and $D_{r}$ are clearly separable. Over this range, if purely translational motions are evident, the decay modes due to $D_{t}$ will all collapse. Eq. (10) can be applied to these angles, obtaining q-independent decay rates. The extracted $D_{t}$ can be averaged, and used as an input to the DDLS data to obtain $D_{r}$.

\section{Experimental}

Gold nanorods of four different aspect ratios were purchased from Nanopartz (Loveland CO). Reported aspect ratios are based on manufacturer's specification measured via transmission electron microscope (TEM). Rod measurements are as follows (long diameter (nm) - axial diameter (nm) - aspect ratio). Rod8.1(565 - 69 - 8.1), Rod2.9(118 - 40 - 2.95), Rod1.7(69 - 40 - 1.73) and Rod4.1(41 - 10 - 4.1). Experiments were performed on an ALV-5022F light scattering spectrometer with a laser wavelength of $633 \mathrm{~nm}$. Samples were suspended in water with $<100 \mathrm{mM}$ Cetrimonium bromide (CTAB) as stabilising agent and measured in a cylindrical glass cuvette (Inner diamater $8 \mathrm{~mm}$ ) (LSI Instruments, Fribourg) held in a scattering vat temperature controlled to $20^{\circ} \mathrm{C}$ (Thermo Neslab RTE 10). A Glan-Thompson

prism (extinction ratio $>10^{5}$ ) was used to select the polarization of the scattered light. DLS measurements of $120 \mathrm{~s}$ duration were obtained in $\mathrm{I}_{\mathrm{VV}}$ and $\mathrm{I}_{\mathrm{VH}}$ modes at scattering angles from $12^{\circ}$ to $140^{\circ}$ with an angular step of $2^{\circ}$. The data is processed with a custom graphic user interface written in MATLAB (Mathworks, Natick, NA). Curve fitting is done using MATLABs least-squares fitting algorithm, and fit quality is checked with the normalized root mean square error (NRMSE) option of MATLABs goodness of fit function. The NRMSE have been provided (where 1 = perfect fit) in the figure captions where fits are shown. 


\section{Results}

\section{Data Processing example with Rod4.1}

We present a protocol that makes uses of data visualization to extract $D_{t}$ and $D_{r}$ of rod-like particles using $V V$ and $V H$ multi angle DLS. Below we illustrate the method for Rod4.1, and show examples for the other three samples in supplementary material.

Firstly, correlation functions $g^{(2)}(\mathbf{q}, \tau)$ are recorded in both $V V$ and $V H$ modes over a range of scattering angles $\left(12^{\circ}\right.$ to $\left.140^{\circ}\right) \cdot g^{(1)}(\mathbf{q}, \tau)$ is obtained from $g^{(2)}(\mathbf{q}, \tau)$ calculated from Eq. (6) after determining $\beta$ by averaging the first 20 points $(V V)$ or 10 points $(V H)$ in order to enable visualisation.

The $V V$ EACFs typically display a single or double decay mode, depending on whether

or not the intensity fluctuation time scales from different motions are separable. These processes could include translational diffusion perpendicular or parallel to the symmetry axis, rotational diffusion, or a combination of both. $V V$ EACFs of Rod4.1 are shown in Fig. 2 whereby two distinct decays are apparent with the shoulder (transition from the first decay to the second) being more pronounced at lower q. In order to gain insight as to what physical processes may be behind the decays of the multi-angle EACFs, we turn to the $\mathbf{q}^{2} \tau$ scaled data.

In Fig. 3a, the entire range of data from $12^{\circ}$ to $140^{\circ}$ is plotted as a function of $\mathbf{q}^{2} \tau$. The scaling of the EACFs allows visualisation of curves that superimpose when plotted together. For spherical optically isotropic diffusive scatterers, the EACF will be a single exponential decay, and curves collected across the entire range of scattering angles will collapse onto a single 'master curve'. In $V V$ mode, if the EACF is a combination of $D_{t}$ and $D_{r}$, a collapse will only be evident over the range of angles where the decay is purely from $D_{t}$. Two features stand out when plotting the data against $\mathbf{q}^{2} \tau$. The first decay shows no apparent scaling across all scattering angles, and is likely a combination of translational and rotational diffusion. However, it is unclear how much each process contributes to the decay of the 
EACF. If only geometric properties of the rods are considered, then Fig. 1 (generated from Eq. (9)) suggests that in $V V$ mode, the lower angles should be dominated by translational motion (the $S_{0}$ term) giving a single exponential decay. The emergence of a clear double decay across all scattering angles highlights the importance of $\mathbf{q}^{2} \tau$ scaling to check which range of angles $D_{t}$ may be obtained from.

The second decay on the other hand shows a clear scaling across the higher scattering angles, indicating that detected motions agree with theory of $D_{t}$. EACFs are removed until only those that collapse at the second decay remain, demonstrated in Fig. 3b. These EACFs are fitted with Eq. 10, and over delay times where the curves superimpose, the resulting decay rates can be averaged to give a value of $D_{t}$.

Fig. 4 illustrates two fits to the EACFs that show scaling. Fitting is performed on $g^{(1)}(\tau)$ with the intercept as a fitting parameter. The shoulder is much more pronounced at low scattering angle, as the time scales between the faster rotational and slower diffusive processes are more separable. The diffusion coefficients calculated from all the ISFs are shown in Fig. 5, which highlights that the curves that do not scale lead to inconsistent values of $D_{t}$. However, consistent results are obtained from the EACFs that collapse, confirming that the $D_{t}$ measured is purely from translational motions.

The $V H$ EACFs are shown in Fig. 6, and appear to match up almost perfectly across all scattering angles showing one clear decay. The $V H$ data contains contributions from both $D_{t}$ and $D_{r}$, but as $D_{r}$ is not dependant on scattering angle, the $D_{t}$ value obtained from the $V V$ data can be used as an input. The data is fitted with Eq. 13 and $D_{r}$ is calculated as $27804 \mathrm{~s}^{-1}$. An example of a fitted $V H$ EACF is shown in Fig. 7. The fit is good considering no polydispersity is accounted for in Eq. (10) and (13), which are often applied in DDLS studies. As the rods have a very low (but non-zero) polydispersity, including a distribution of both $D_{t}$ and $D_{r}$ is beyond the scope of this paper. 


\section{Comparing DLS results to theory}

The results obtained from this method are summarised in table 1 and compared with the theoretical results. For the two largest nanorods (Rod8.1 and Rod2.9), the TG theory shows good agreement with the experimental results, whereas the BR theory significantly underestimates $D_{t}(\operatorname{Rod} 8.1)$ and $D_{r}(\operatorname{Rod} 2.9)$.

For Rod1.7, the TG theory again shows good agreement. The aspect ratio for this sample is below where the BR theory should apply, so it is not compared.

Despite good fits for Rod4.1, values of $D_{r}$ and $D_{t}$ were overestimated by both theories. However, experimental values agree well to a spheroidal model, ${ }^{29}$ which resulted in

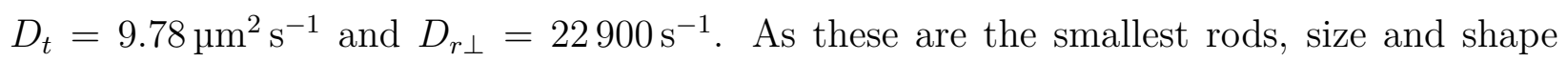
polydispersity as well as the relative contribution of the stabilisation layer have a much larger impact on dynamics than for bigger rods, so the spheroidal approximation is probably closer to the real shape of the rods in solution.

The EACFs from Rod8.1 were in fact the most difficult to analyse as they did not display a clear double exponential decay (see supplementary material Fig. S1(C)). They were fitted with the single exponential decay of Eq. 4, and $D_{t}$ was obtained from $70^{\circ}$ to $140^{\circ}$ where scaling was best. This again raises questions as to the range of L and D for which Eq. 9 is applicable and what effect the optical anisotropy has on top of this, as the form factor of Rod8.1 should introduce rotational contributions from around $\theta=70^{\circ}$. The $V H$ data from Rod8.1 was also interesting as it does not overlap. When calculating $D_{r}$ from the $V H$ EACFs from Rod8.1, consistent results were found from $\theta=12^{\circ}$ to $80^{\circ}$. Above this angle, the values become nonsensical. It is likely that this is due to the form factor approaching its minimum and therefore affecting the contribution of $D_{t}$. 
Table 1: Summary of the nanorods used, and comparisons of DDLS measured values with theory. The percentage are the discrepancies between the theoretical and our experimental results. Units of $D_{t}$ are $\mu \mathrm{ms}^{-2}$ and $D_{r}$ are $\mathrm{s}^{-1}$.

\begin{tabular}{|c|c|c|c|c|}
\hline $\begin{array}{l}\text { Specification } \\
(\mathrm{L}, \mathrm{D})(\mathrm{nm})\end{array}$ & $\begin{array}{l}\text { Aspect } \\
\text { Ratio } \\
\text { (L/D) }\end{array}$ & $\begin{array}{l}\text { Tirado and Gar- } \\
\text { ica de la Torre }\end{array}$ & Broersma & DDLS calculated \\
\hline Rod8.1565 69 & 8.1 & & & \\
\hline$D_{t}$ & & $1.88(-29.32 \%)$ & $\begin{array}{l}1.56 \\
(-41.35 \%)\end{array}$ & 2.66 \\
\hline$D_{r}$ & & $33.2(1.53 \%)$ & $\begin{array}{l}30.06 \\
(-8.07 \%)\end{array}$ & 32.7 \\
\hline Rod2.9 11840 & 2.95 & & & \\
\hline$D_{t}$ & & $5.71(12.62 \%)$ & $\begin{array}{l}4.76 \\
(-6.11 \%)\end{array}$ & 5.07 \\
\hline$D_{r}$ & & $1714(-6.24 \%)$ & $\begin{array}{l}1087 \\
(-40.54 \%)\end{array}$ & 1828 \\
\hline Rod1.7 6940 & 1.73 & & & \\
\hline$D_{t}$ & & $7.15(13.67 \%)$ & & 6.29 \\
\hline$D_{r}$ & & $4852(17.97 \%)$ & & 4113 \\
\hline Rod4.1 4110 & 4.1 & & & \\
\hline$D_{t}$ & & $19.4(101.87 \%)$ & $\begin{array}{l}15.1 \\
(47858 \%)\end{array}$ & 9.61 \\
\hline$D_{r}$ & & $54282(95.23 \%)$ & $\begin{array}{l}47858 \\
(72.13 \%)\end{array}$ & 27804 \\
\hline
\end{tabular}




\section{Conclusion}

We have presented a new analysis protocol for measuring nano rods using DDLS. The method ensures that purely translational diffusive motions are obtained from the vertically polarized scattering data by visualisation of $\mathbf{q}^{2} \tau$ scaling. For the systems that display two decay modes (Rod2.9, Rod1.7, Rod4.1), the angles for which translational diffusion dominates are easily identifiable, allowing the calculation of $D_{t}$. For Rod8.1, which displayed only a single decay mode, scattering data at higher angles returns a consistent $D_{t}$, while lower angles are required to calculate $D_{r}$.

The existing theoretical models have not been thoroughly experimentally tested, due to ambiguities in the interpretation of the scattering data. The method proposed here overcomes these difficulties, and diffusion coefficients obtained from this method show good agreement with calculations based on the direct measurements of L and D. Further studies over a wider range of particle sizes, materials, and aspect ratios are needed to further examine the range of validity of these theories.

Moreover, for rods with dimensions where the theories are shown to be valid, the method proposed here will allow the measurement of statistically meaningful particle sizes and aspect ratios. This will complement real space methods, which provide high resolution but where statistical averaging is necessarily limited. 


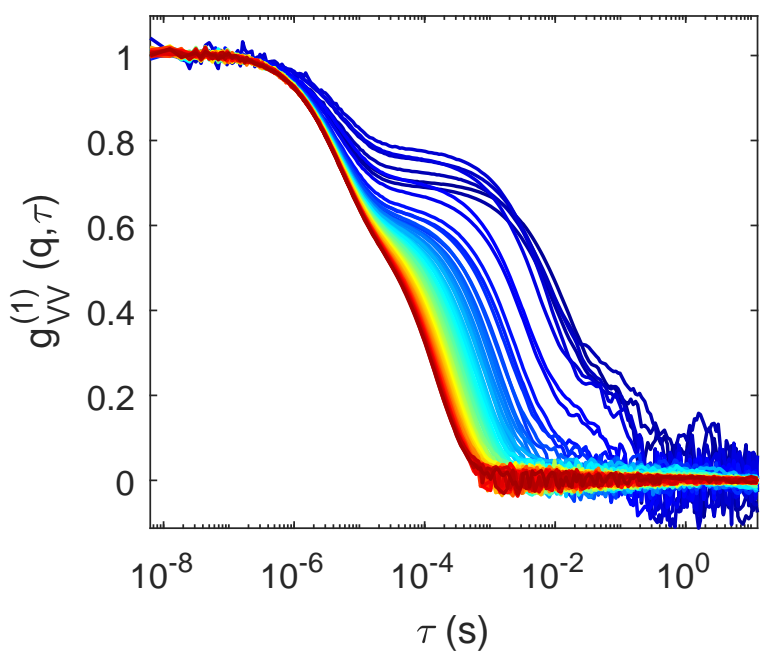

Figure 2: $V V$ EACFs for Rod4.1 taken from $12^{\circ}$ to $140^{\circ}$ at $2^{\circ}$ steps. The angles increase from the blue to the red end of the colour spectrum. 

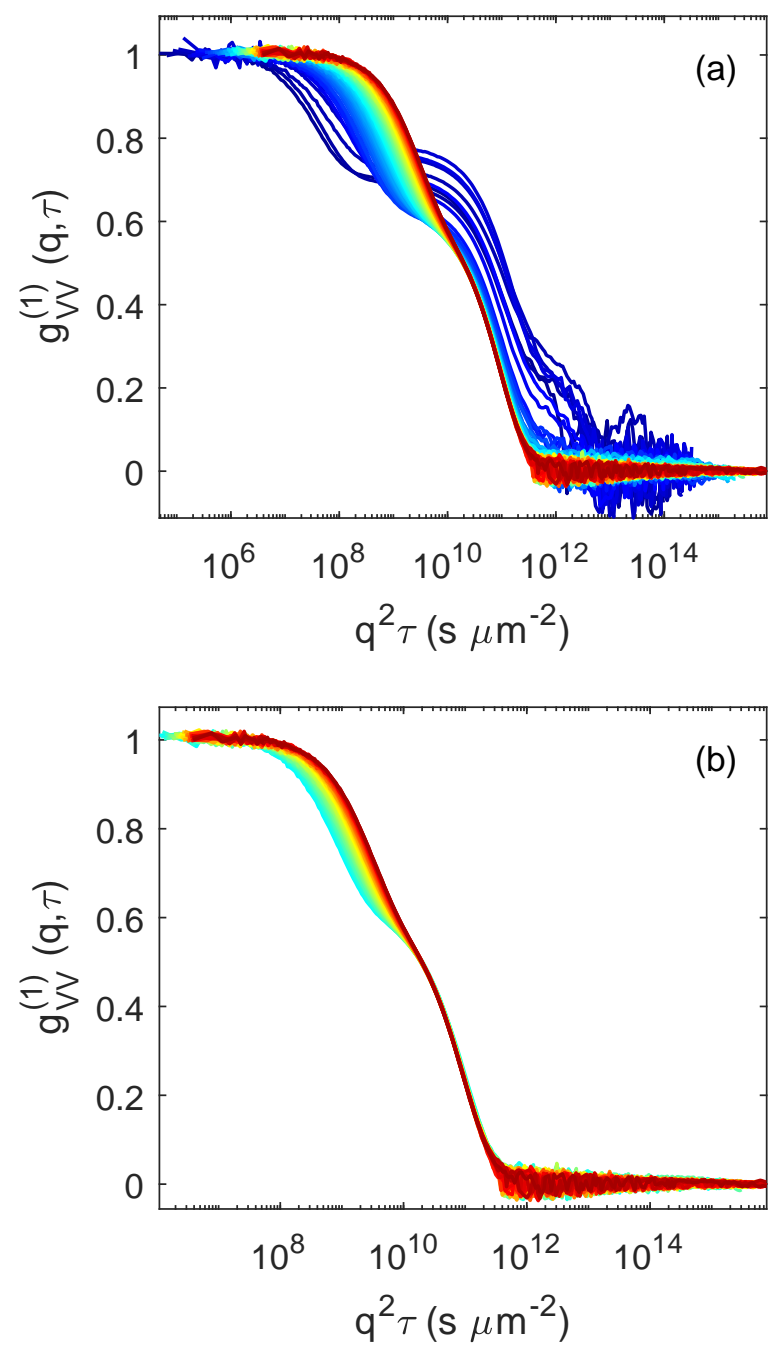

Figure 3: (a) $V V$ EACFs of Rod4.1 plotted against $\mathbf{q}^{2} \tau$. (b) The same data as (a), but with curves that do not collapse at the second decay removed. Data that remains is from $\theta=60^{\circ}$ to $140^{\circ}$. 


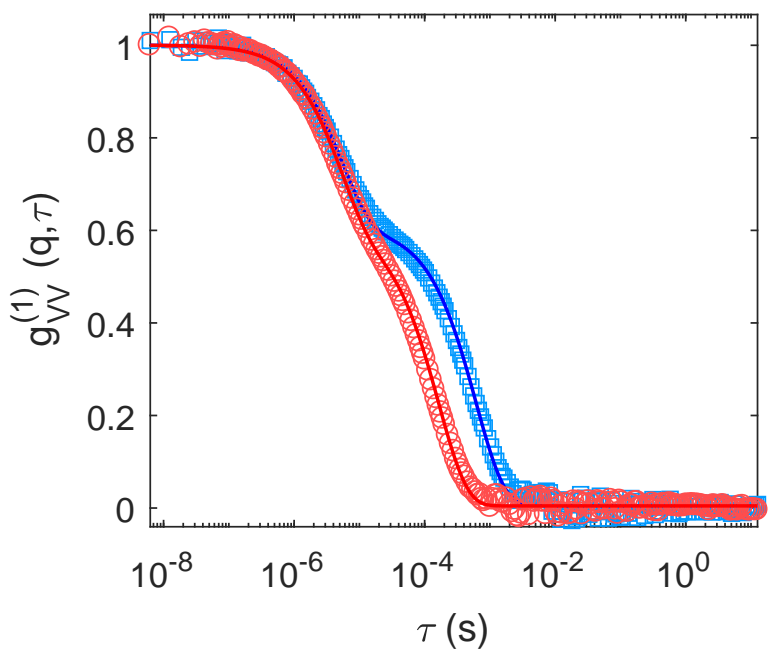

Figure 4: Raw data (symbols) and fits (line) for lowest (blue squares, $\theta=60^{\circ}, N R M S E=$ 0.97 ) and highest (red circles, $\theta=140^{\circ}, N R M S E=0.98$ ) scattering angles that scale from Fig. 3 (b).

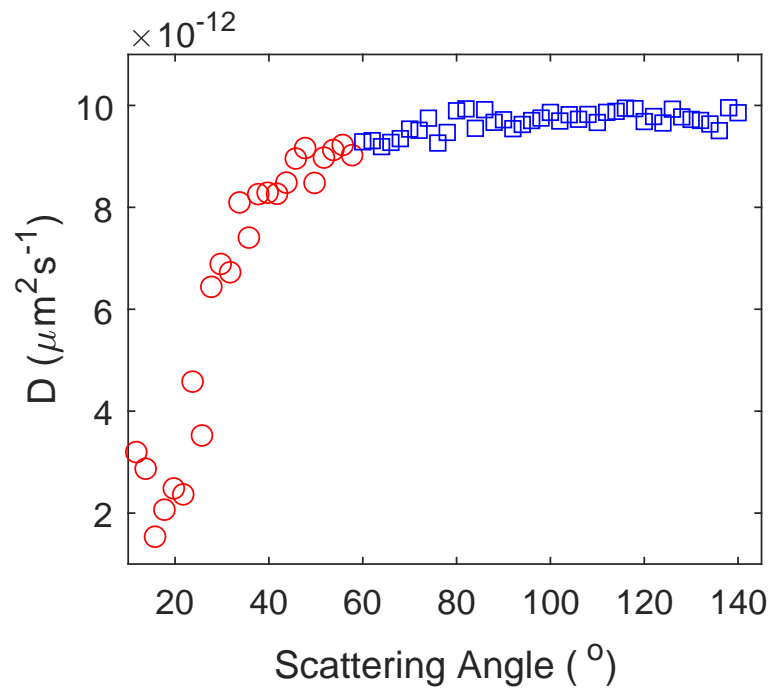

Figure 5: Fitted $D_{t}$ using Eq. 10 for curves that do not collapse (red circles), and the curves that do (blue squares). The curves that collapse in accordance with $\mathbf{q}^{2} \tau$ return a consistent result for $D_{t}$ of average $9.61 \mathrm{\mu m}^{2} \mathrm{~s}^{-1}$ 


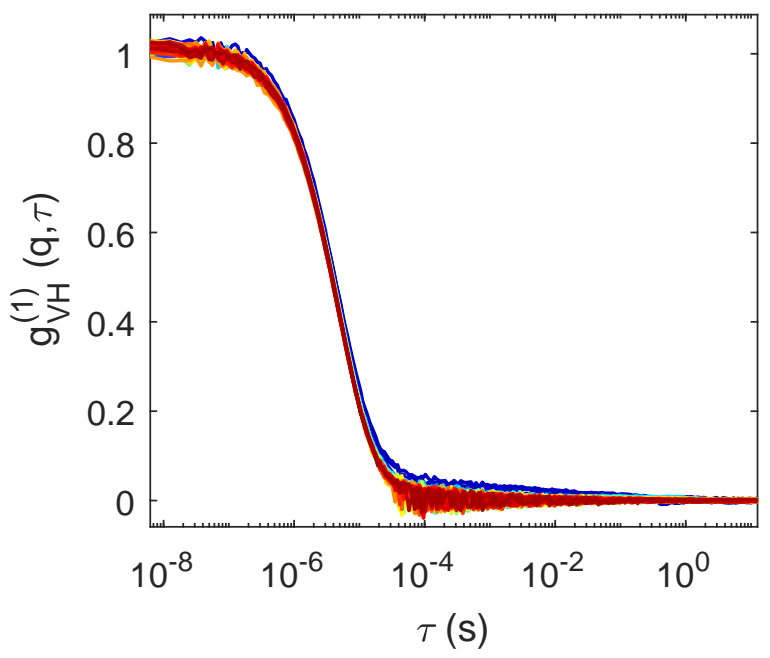

Figure 6: $V H$ EACFs for Rod4.1 taken $12^{\circ}$ to $140^{\circ}$ at $2^{\circ}$ steps. The angles increase from the blue to the red end of the spectrum.

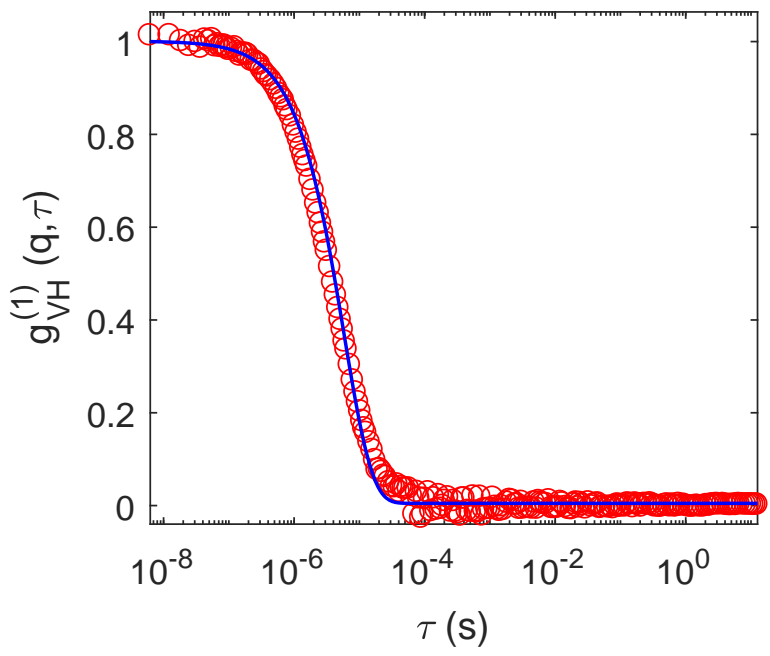

Figure 7: $V H$ EACF at $\theta=90^{\circ}$ (red circles) and fit (blue line, $N R M S E=0.96$ ) with Eq. 13 using $D_{t}=9.61 \mathrm{\mu m}^{2} \mathrm{~s}^{-1}$. Calculated $D_{r}=27804 \mathrm{~s}^{-1}$. 


\section{Supporting Information Available}

Supporting Information is available from the Wiley Online Library: Plots for the entire

DDLS protocol for Rod1.7, Rod2.9 and Rod8.1. TEM images of Rod1.7 and Rod8.1. 


\section{References}

(1) Orendorff, C. J.; Gearheart, L.; Jana, N. R.; Murphy, C. J. Aspect ratio dependence on surface enhanced Raman scattering using silver and gold nanorod substrates. Physical Chemistry Chemical Physics 2006, 8, 165-170.

(2) Carnovale, C.; Bryant, G.; Shukla, R.; Bansal, V. Size, shape and surface chemistry of nano-gold dictate its cellular interactions, uptake and toxicity. Progress in Materials Science 2016, 83, 152-190.

(3) Lehner, D.; Lindner, H.; Glatter, O. Determination of the translational and rotational diffusion coefficients of rodlike particles using depolarized dynamic light scattering. Langmuir 2000, 16, 1689-1695.

(4) van der Zande, B. M.; Dhont, J. K.; Böhmer, M. R.; Philipse, A. P. Colloidal dispersions of gold rods characterized by dynamic light scattering and electrophoresis. Langmuir 2000, 16, 459-464.

(5) Koenderink, G. H.; Philipse, A. P. Rotational and translational self-diffusion in colloidal sphere suspensions and the applicability of generalized Stokes-Einstein relations. Langmuir 2000, 16, 5631-5638.

(6) Geers, C.; Rodriguez-Lorenzo, L.; Urban, D. A.; Kinnear, C.; Petri-Fink, A.; Balog, S. A new angle on dynamic depolarized light scattering: number-averaged size distribution of nanoparticles in focus. Nanoscale 2016, 8, 15813-15821.

(7) Balog, S.; Rodriguez-Lorenzo, L.; Monnier, C. A.; Michen, B.; Obiols-Rabasa, M.; Casal-Dujat, L.; Rothen-Rutishauser, B.; Petri-Fink, A.; Schurtenberger, P. Dynamic depolarized light scattering of small round plasmonic nanoparticles: when imperfection is only perfect. The Journal of Physical Chemistry C 2014, 118, 17968-17974. 
(8) Shetty, A. M.; Wilkins, G. M.; Nanda, J.; Solomon, M. J. Multiangle depolarized dynamic light scattering of short functionalized single-walled carbon nanotubes. The Journal of Physical Chemistry C 2009, 113, 7129-7133.

(9) Khouri, S.; Shams, M.; Tam, K. C. Determination and prediction of physical properties of cellulose nanocrystals from dynamic light scattering measurements. Journal of nanoparticle research 2014, 16, 1-14.

(10) Balog, S.; Rodriguez-Lorenzo, L.; Monnier, C. A.; Obiols-Rabasa, M.; RothenRutishauser, B.; Schurtenberger, P.; Petri-Fink, A. Characterizing nanoparticles in complex biological media and physiological fluids with depolarized dynamic light scattering. Nanoscale 2015, 7, 5991-5997.

(11) Bossert, D.; Natterodt, J.; Urban, D. A.; Weder, C.; Petri-Fink, A.; Balog, S. Specklevisibility spectroscopy of depolarized dynamic light scattering. The Journal of Physical Chemistry B 2017, 121, 7999-8007.

(12) Liu, T.; Xiao, Z. Dynamic light scattering of rigid rods-a universal relationship on the apparent diffusion coefficient as revealed by numerical studies and its use for rod length determination. Macromolecular Chemistry and Physics 2012, 213, 1697-1705.

(13) Berne, B. J.; Pecora, R. Dynamic light scattering: with applications to chemistry, biology, and physics; Courier Corporation, 1976.

(14) Rodríguez-Fernández, J.; Pérez-Juste, J.; Liz-Marzán, L. M.; Lang, P. R. Dynamic light scattering of short Au rods with low aspect ratios. The Journal of Physical Chemistry C 2007, 111, 5020-5025.

(15) Borsali, R.; Pecora, R. Soft-matter characterization; Springer Science \& Business Media, 2008. 
(16) Ferri, F.; Magatti, D.; Pescini, D.; Potenza, M.; Giglio, M. Heterodyne near-field scattering: A technique for complex fluids. Physical Review E 2004, 70, 041405.

(17) Escobedo-Sánchez, M. A.; Hugo, A.; Arauz-Lara, J. L.; Haro-Pérez, C.; RojasOchoa, L. F. Study of translational and rotational dynamics of birefringent colloidal particles by depolarized light scattering in the far-and near-field regimes. The Journal of chemical physics 2015, 143, 044902.

(18) Brogioli, D.; Salerno, D.; Cassina, V.; Sacanna, S.; Philipse, A. P.; Croccolo, F.; Mantegazza, F. Characterization of anisotropic nano-particles by using depolarized dynamic light scattering in the near field. Optics express 2009, 17, 1222-1233.

(19) Glidden, M.; Muschol, M. Characterizing Gold Nanorods in Solution Using Depolarized Dynamic Light Scattering. The Journal of Physical Chemistry C 2012, 116, 8128-8137.

(20) Potenza, M.; Sanvito, T.; Alaimo, M.; Degiorgio, V.; Giglio, M. Confocal zero-angle dynamic depolarized light scattering. The European Physical Journal E 2010, 31, 69-72.

(21) Cerbino, R.; Trappe, V. Differential dynamic microscopy: probing wave vector dependent dynamics with a microscope. Physical review letters 2008, 100, 188102.

(22) Chen, S.-H.; Hallett, F. R. Determination of motile behaviour of prokaryotic and eukaryotic cells by quasi-elastic light scattering. Quarterly reviews of biophysics $\mathbf{1 9 8 2}$, $15,131-222$.

(23) Johnson, C.; Gabriel, D. Laser Light Scattering; Dover Books on Physics; Dover Publications, 1994.

(24) Dhont, J. K. An introduction to dynamics of colloids; Elsevier, 1996; Vol. 2.

(25) Wang, C. H. Spectroscopy of condensed media: dynamics of molecular interactions; Elsevier, 2012. 
(26) Tirado, M. M.; de la Torre, J. G. Translational friction coefficients of rigid, symmetric top macromolecules. Application to circular cylinders. The Journal of Chemical Physics 1979, 71, 2581-2587.

(27) Tirado, M. M.; de la Torre, J. G. Rotational dynamics of rigid, symmetric top macromolecules. Application to circular cylinders. The Journal of Chemical Physics 1980, 73, 1986-1993.

(28) Broersma, S. Viscous force constant for a closed cylinder. The Journal of Chemical Physics 1960, 32, 1632-1635.

(29) Hoffmann, M.; Wagner, C. S.; Harnau, L.; Wittemann, A. 3D Brownian diffusion of submicron-sized particle clusters. ACS nano 2009, 3, 3326-3334. 


\title{
Supplementary Information
}

\section{A Depolarized Dynamic Light Scattering Method to Calculate Translational and Rotational Diffusion Coefficients of Nanorods}

\author{
Mr. Reece Nixon-Luke* and Prof. Gary Bryant \\ Centre for Molecular and Nanoscale Physics, School of Science, RMIT University, \\ Melbourne, Victoria 3001, Australia \\ E-mail: reece.nxion-luke@rmit.edu.au
}



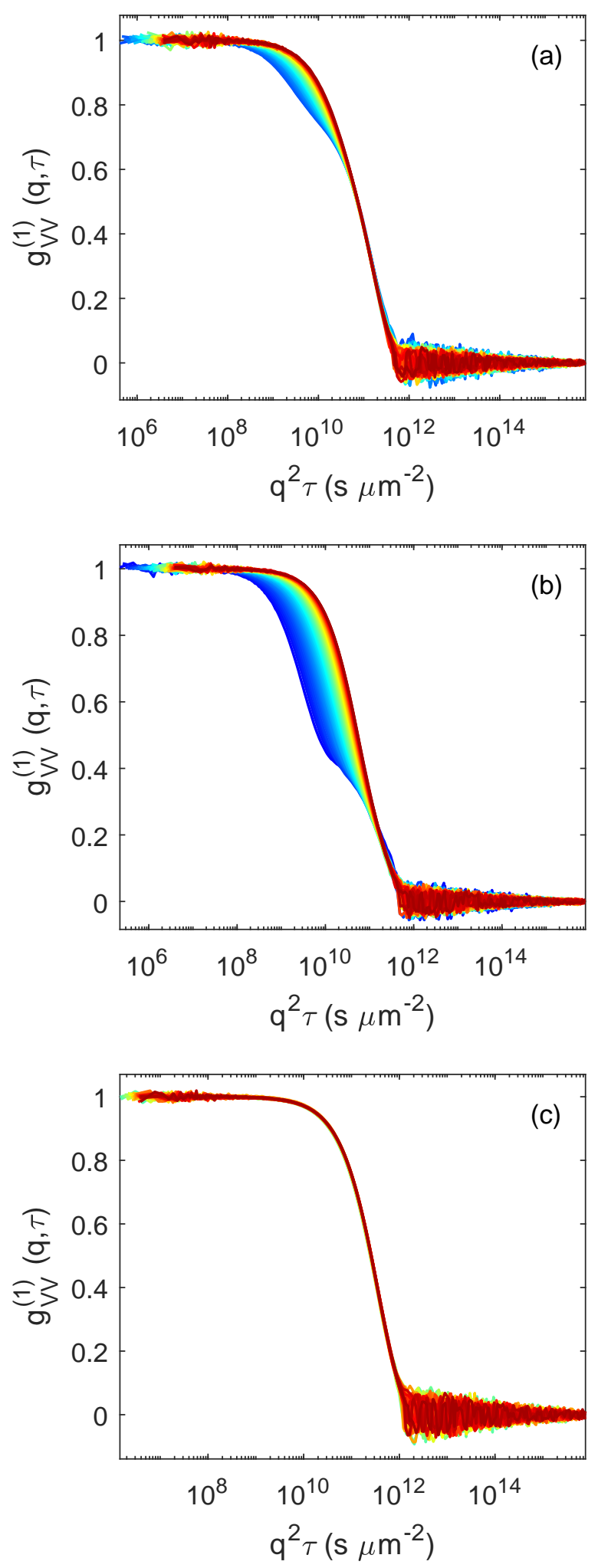

Figure S1: $V V$ EACFs that conform to scaling plotted against $\mathbf{q}^{2} \tau$ for three rods. (a) Rod1.7 $\theta=36^{\circ}$ to $140^{\circ}$, (b) $\operatorname{Rod} 2.9 \theta=26^{\circ}$ to $140^{\circ}$, (c) $\operatorname{Rod} 8.1 \theta=70^{\circ}$ to $140^{\circ}$. The angles increase from the red to the blue end of the spectrum at $2^{\circ}$ intervals. 

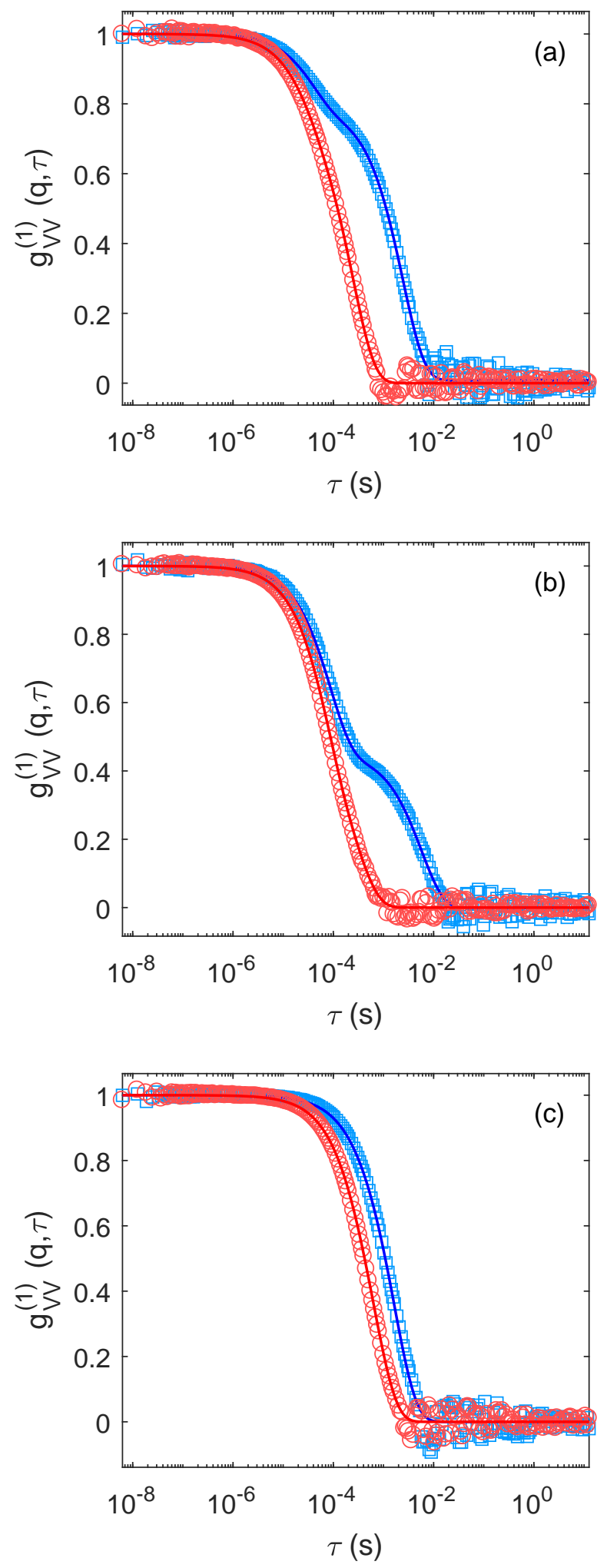

Figure S2: Raw data (symbols) and fits (line) for lowest (blue squares) and highest (red circles) scattering angles displayed in Fig. S1. 

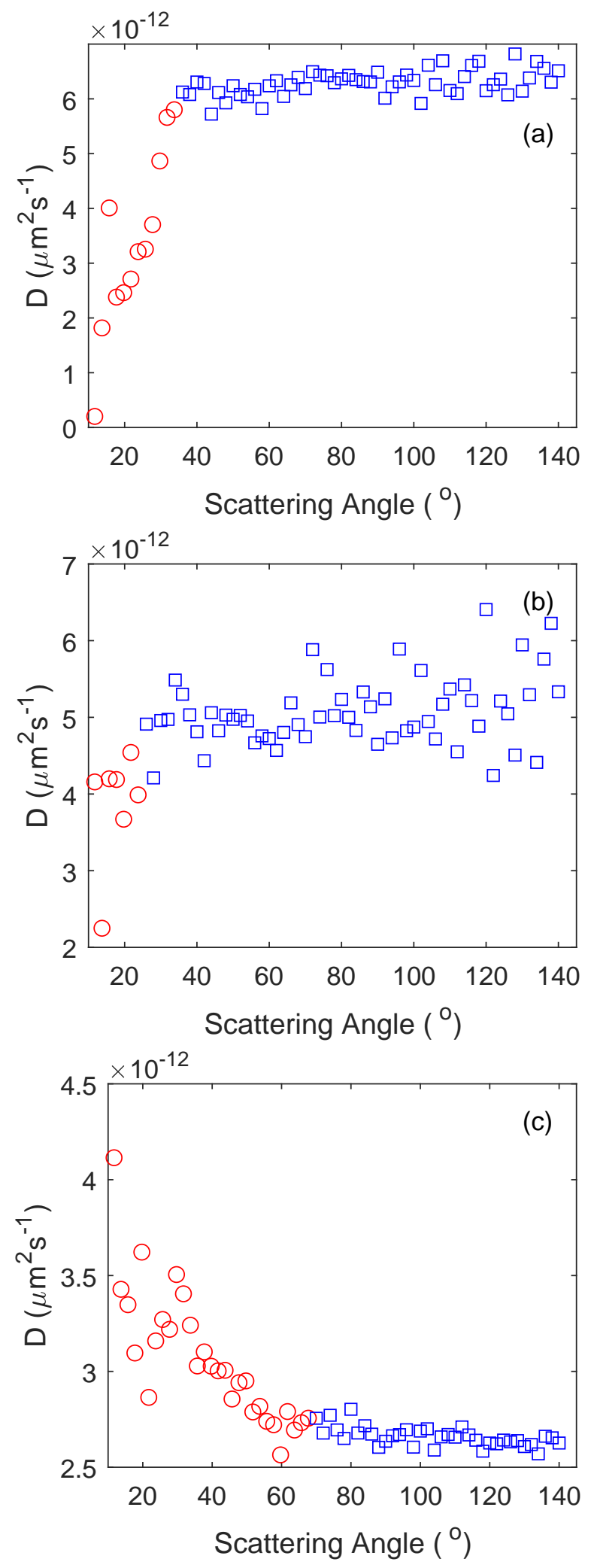

Figure S3: Fitted $D_{t}$ values for curves that do not collapse (red circles), and the curves that do collapse (blue squares), as displayed in Fig. S1. Resulting $D_{t}$ 's are (a) Rod1.7

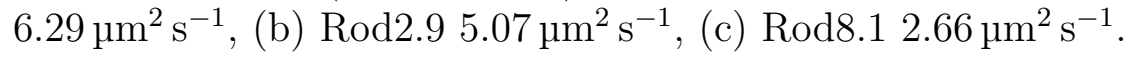



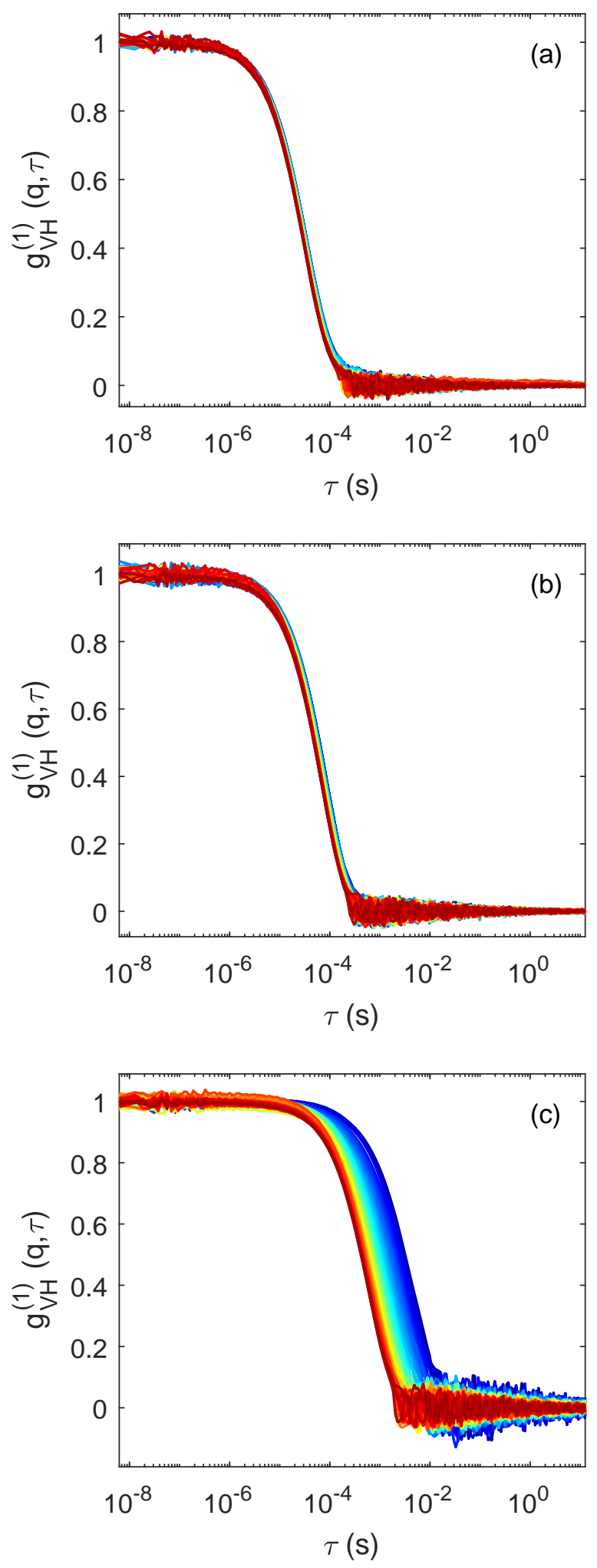

Figure S4: $V H$ EACFs for (a) $\operatorname{Rod} 1.7 \theta=12^{\circ}$ to $140^{\circ}$, (b) $\operatorname{Rod} 2.9 \theta=12^{\circ}$ to $140^{\circ}$, (c) Rod8.1 $\theta=12^{\circ}$ to $80^{\circ}$. The angles increase from the red to the blue end of the spectrum at $2^{\circ}$ intervals. 

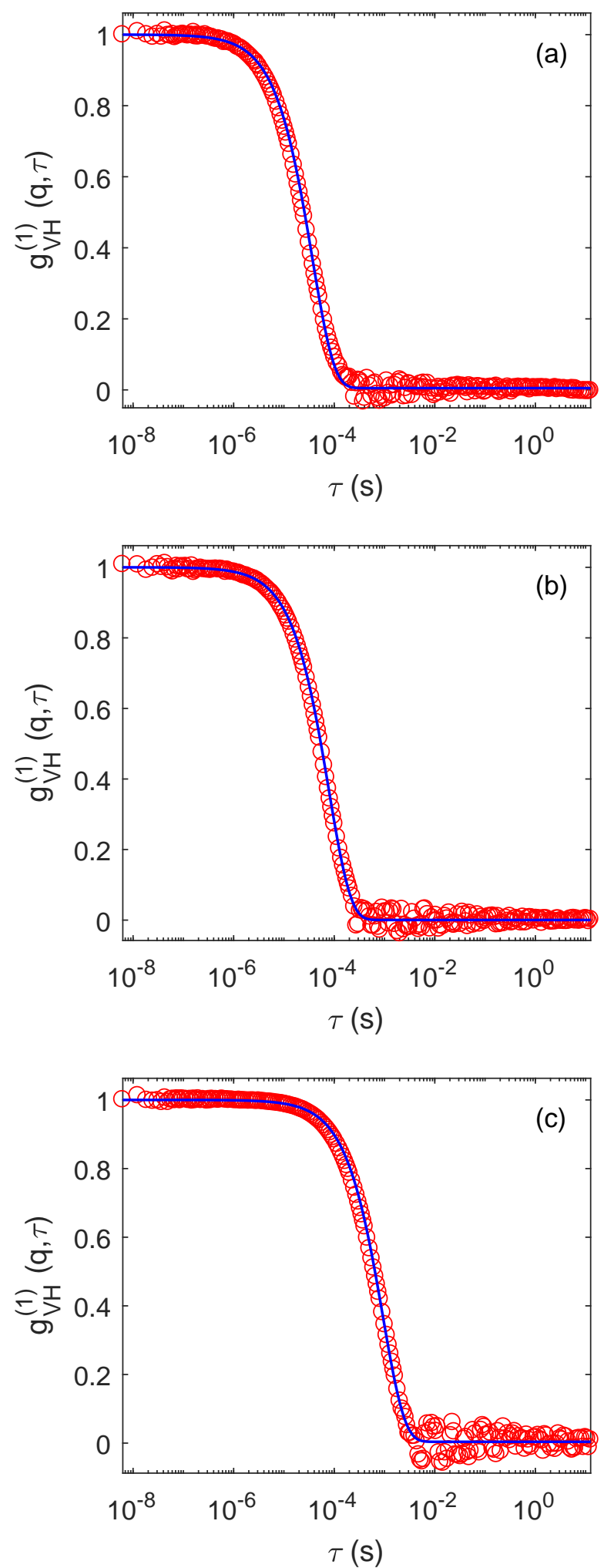

Figure S5: $V H$ EACFs (red circles) and fit (blue line) for (a) Rod1.7 $\theta=90^{\circ}$, (b) $\operatorname{Rod} 2.9$ $\theta=90^{\circ}$, (c) $\operatorname{Rod} 8.1 \theta=50^{\circ}$. 

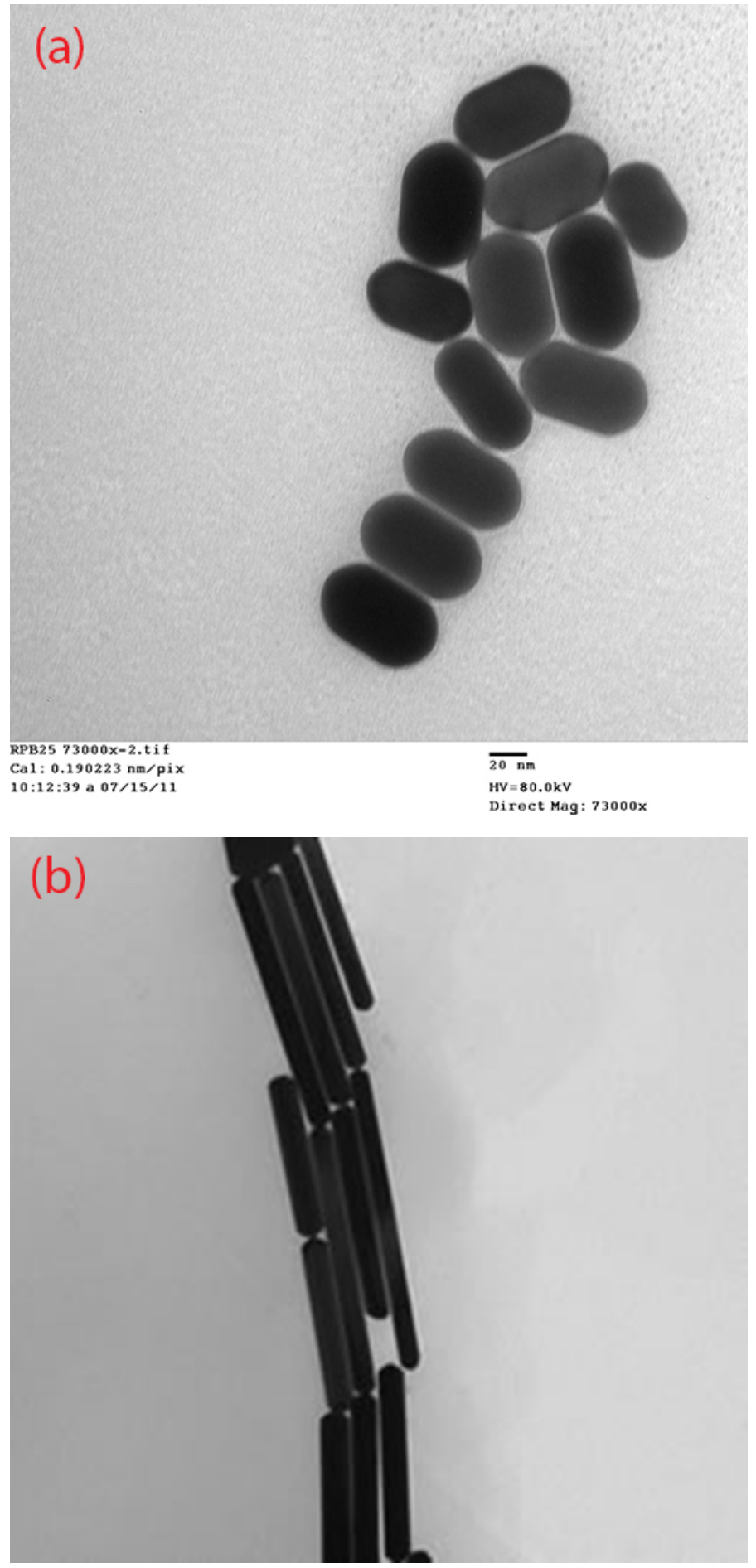

Figure S6: Transmission Electron Microscope images (obtained from Nanopartz) showing polydispersity for (a) Rod1.7 and (b) Rod8.1 